\title{
On the maximum likelihood estimators for parameters of a Weibull distribution under random censoring
}

\author{
Namhyun $\mathrm{Kim}^{1, a}$ \\ ${ }^{a}$ Department of Science, Hongik University, Korea
}

\begin{abstract}
In this paper, we consider statistical inferences on the estimation of the parameters of a Weibull distribution when data are randomly censored. Maximum likelihood estimators (MLEs) and approximate MLEs are derived to estimate the parameters. We consider two cases for the censoring model: the assumption that the censoring distribution does not involve any parameters of interest and a censoring distribution that follows a Weibull distribution. A simulation study is conducted to compare the performances of the estimators. The result shows that the MLEs and the approximate MLEs are similar in terms of biases and mean square errors; in addition, the assumption of the censoring model has a strong influence on the estimation of scale parameter.

Keywords: approximate maximum likelihood estimators, Koziol-Green model, maximum likelihood estimators, power comparison, random censoring, Weibull distribution
\end{abstract}

\section{Introduction}

In survival studies, data may be subject to random censoring. Let $T_{1}, \ldots, T_{n}$ denote lifetimes with distribution function $F$ and probability density function (pdf) $f$. The censoring times $C_{1}, \ldots, C_{n}$ drawn independently of the $T_{i}$ are from distribution function $G$ and pdf $g$. The $T_{i}$ 's are censored on the right by $C_{i}$, so we observe $n$ i.i.d. random pairs $\left(X_{j}, \delta_{j}\right), j=1, \ldots, n$, where

$$
X_{j}=\min \left(T_{j}, C_{j}\right) \quad \text { and } \quad \delta_{j}= \begin{cases}1, & \text { if } X_{j}=T_{j}, \\ 0, & \text { if } X_{j}=C_{j} .\end{cases}
$$

For a model of random censorship, Koziol and Green (1976) introduced a special model with

$$
1-G=(1-F)^{\beta}, \quad \text { for some } \beta>0 .
$$

Under this model, we have

$$
P\left(T_{i}>C_{i}\right)=\int_{-\infty}^{\infty}(1-F(x)) d G(x)=\int_{0}^{1} \beta(1-x)^{\beta} d x=\frac{\beta}{\beta+1} .
$$

Hence, $\beta /(\beta+1)$ is the expected proportion of the censored observations and $\beta$ is called the censoring parameter. The case $\beta=0$ corresponds to no censoring. Csörgó and Horváth (1981) called (1.2) the Koziol-Green model of random censorship. The motivation and characterization of this model is discussed in Chen et al. (1982) and Kim (2014).

\footnotetext{
${ }^{1}$ Department of Science, Hongik University, 94 Wausan-Ro, Mapo-Gu, Seoul 04066, Korea. E-mail: nhkim@ @ongik.ac.kr

Published 31 May 2016/ journal homepage: http://csam.or.kr

(c) 2016 The Korean Statistical Society, and Korean International Statistical Society. All rights reserved.
} 
Suppose the lifetime distribution follows a Weibull distribution $\operatorname{Weibull}(\lambda, \alpha)$ with the distribution function

$$
F(x)=1-e^{-\lambda x^{\alpha}}, \quad x>0
$$

and the pdf

$$
f(x)=\alpha \lambda x^{\alpha-1} e^{-\lambda x^{\alpha}}, \quad x>0,
$$

where $\lambda$ and $\alpha$ are the scale and shape parameters, respectively, and are positive.

This paper derives the maximum likelihood estimators (MLEs) and the approximate MLEs of the parameters of a Weibull distribution under random censoring. The analysis of censored data usually assumes that the censoring distribution does not involve any parameters of interest. We find the MLEs and the approximate MLEs in this case and those under the Koziol-Green model in (1.2) to compare them. In Section 2, we obtain the MLEs of the parameters of a Weibull distribution. We also derive the approximate MLEs using the type I extreme value distribution of the minimum since the MLEs cannot be expressed in explicit form. In Section 3, we conduct a simulation study to compare the MLEs and the approximate MLEs. We generate censored random samples under the Koziol-Green model and compute the estimators in two different ways. One is the estimators ignoring the Koziol-Green model (assuming the censoring distribution does not involve any parameters) and the other is the estimators under the true model. We compare the two of them to see what happens when we ignore the random censorship model. Section 4 ends the paper with some concluding remarks.

\section{MLEs and the approximate MLEs}

\subsection{MLEs and the approximate MLEs under the assumption that the censoring distribution does not involve any parameters}

For the observed random pairs $\left(X_{j}, \delta_{j}\right)$ in $(1.1)$, we assume that $X_{j}$ 's are the ordered observations without loss of generality. The likelihood function based on $\left(X_{j}, \delta_{j}\right)$ is given by (Tableman and Kim, 2003)

$$
\begin{aligned}
L & =n ! \prod_{i=1}^{n}\left(f\left(x_{i}\right) \bar{G}\left(x_{i}\right)\right)^{\delta_{i}}\left(g\left(x_{i}\right) \bar{F}\left(x_{i}\right)\right)^{1-\delta_{i}} \\
& =n ! \prod_{i=1}^{n} f\left(x_{i}\right)^{\delta_{i}} \bar{F}\left(x_{i}\right)^{1-\delta_{i}} \prod_{i=1}^{n} g\left(x_{i}\right)^{1-\delta_{i}} \bar{G}\left(x_{i}\right)^{\delta_{i}},
\end{aligned}
$$

where $\bar{F}=1-F, \bar{G}=1-G$. If we assume that the distribution of the censoring time $C_{i}$ 's does not involve any parameters of interest, the part $\prod_{i=1}^{n} g\left(x_{i}\right)^{1-\delta_{i}} \bar{G}\left(x_{i}\right)^{\delta_{i}}$ in (2.1) plays no role in the estimation process. Hence the likelihood function can be taken to be

$$
L \propto \prod_{i=1}^{n} f\left(x_{i}\right)^{\delta_{i}} \bar{F}\left(x_{i}\right)^{1-\delta_{i}}
$$

If the lifetime distribution follows Weibull $(\lambda, \alpha)$ given in (1.4) or (1.3), then the log-likelihood function $l=\ln L$ becomes

$$
l \propto \sum_{i=1}^{n} \delta_{i}\left[\ln \alpha+\ln \lambda+(\alpha-1) \ln x_{i}-\lambda x_{i}^{\alpha}\right]+\sum_{i=1}^{n}\left(1-\delta_{i}\right)\left(-\lambda x_{i}^{\alpha}\right) .
$$


The MLEs of $\alpha$ and $\lambda$ can be derived by solving the likelihood equations

$$
\begin{aligned}
& \frac{\partial l}{\partial \lambda}=\frac{n_{u}}{\lambda}-\sum_{i=1}^{n} x_{i}^{\alpha}=0, \\
& \frac{\partial l}{\partial \alpha}=\frac{n_{u}}{\alpha}+\sum_{i=1}^{n} \delta_{i} \ln x_{i}-\lambda \sum_{i=1}^{n} x_{i}^{\alpha} \ln x_{i}=0,
\end{aligned}
$$

where $n_{u}=\sum_{i=1}^{n} \delta_{i}$ is the number of uncensored data. From (2.2), we get

$$
\lambda(\alpha)=\frac{n_{u}}{\sum_{i=1}^{n} x_{i}^{\alpha}} .
$$

Substituting back $\lambda(\alpha)$ into (2.3), we obtain the equation of $\alpha$ as

$$
\frac{1}{\alpha}=\frac{\sum_{i=1}^{n} x_{i}^{\alpha} \ln x_{i}}{\sum_{i=1}^{n} x_{i}^{\alpha}}-\frac{1}{n_{u}} \sum_{i=1}^{n} \delta_{i} \ln x_{i} .
$$

Balakrishnan and Kateri (2008) discussed the existence and uniqueness of the MLEs of a Weibull distribution. The equation (2.5) does not have a solution in a compact form and it should be solved in an iterative way.

If the random variable $T$ follows Weibull $(\alpha, \lambda)$, then $\ln T$ follows the type I extreme value distribution of the minimum $\operatorname{EV}(\mu, \sigma)$ with the distribution function and the pdf,

$$
F_{Y}(y)=1-e^{-e^{\frac{y-\mu}{\sigma}}}, \quad f_{Y}(y)=\frac{1}{\sigma} e^{\frac{y-\mu}{\sigma}-e^{\frac{y-\mu}{\sigma}}}, \quad-\infty<y<\infty
$$

where $\mu$ and $\sigma$ are location and scale parameters respectively, and $\mu=-(1 / \alpha) \ln \lambda, \sigma=1 / \alpha$. For the estimation of the extreme value distribution, we refer to Chen (1998) and Engelhardt and Bain (1974).

Pareek et al. (2009) mentioned that $\operatorname{Weibull}(\alpha, \lambda)$ and $\operatorname{EV}(\mu, \sigma)$ are equivalent models because the procedure developed under one model can be used for the other. Nonetheless, we could have an easier estimation process by working with the model $\operatorname{EV}(\mu, \sigma)$ in (2.6) since $\mu$ and $\sigma$ are the location and scale parameters. A similar technique was used by Kundu (2007) and Kang and Han (2009) for different censoring schemes.

Letting $Y_{j}=\ln X_{j}$, the $\log$-likelihood function based on $\left(Y_{j}, \delta_{j}\right)$ becomes

$$
l \propto \sum_{i=1}^{n} \delta_{i}\left[-\ln \sigma+\frac{y_{i}-\mu}{\sigma}-e^{\frac{y_{i}-\mu}{\sigma}}\right]+\sum_{i=1}^{n}\left(1-\delta_{i}\right)\left(-e^{\frac{y_{i}-\mu}{\sigma}}\right) .
$$

Remind that $Z=(Y-\mu) / \sigma$ has a standard extreme value distribution $\operatorname{EV}(0,1)$ with the distribution function and the pdf

$$
F_{Z}(z)=1-e^{-e^{z}}, \quad f_{Z}(z)=e^{z-e^{z}}
$$

when $Y$ follows $\operatorname{EV}(\mu, \sigma)$. Substituting $z_{i}=\left(y_{i}-\mu\right) / \sigma$ in $(2.7)$, we get

$$
l(\mu, \sigma) \propto n_{u}(-\ln \sigma)+\sum_{i=1}^{n} \delta_{i} z_{i}-\sum_{i=1}^{n} e^{z_{i}}
$$


Since $\partial z_{i} / \partial \mu=-(1 / \sigma), \partial e^{z_{i}} / \partial \mu=-(1 / \sigma) e^{z_{i}}, \partial z_{i} / \partial \sigma=-(1 / \sigma) z_{i}$, and $\partial e^{z_{i}} / \partial \sigma=-(1 / \sigma) z_{i} e^{z_{i}}$, we have

$$
\begin{aligned}
& \frac{\partial l}{\partial \mu}=-\frac{n_{u}}{\sigma}+\frac{1}{\sigma} \sum_{i=1}^{n} e^{z_{i}}=0, \\
& \frac{\partial l}{\partial \sigma}=-\frac{n_{u}}{\sigma}-\frac{1}{\sigma} \sum_{i=1}^{n} \delta_{i} z_{i}+\frac{1}{\sigma} \sum_{i=1}^{n} z_{i} e^{z_{i}}=0,
\end{aligned}
$$

by taking derivatives with respect to $\mu$ and $\sigma$ of $l(\mu, \sigma)$ in (2.8). Equivalently

$$
\begin{aligned}
n_{u}-\sum_{i=1}^{n} e^{z_{i}} & =0, \\
n_{u}+\sum_{i=1}^{n} \delta_{i} z_{i}-\sum_{i=1}^{n} z_{i} e^{z_{i}} & =0 .
\end{aligned}
$$

Clearly, (2.9) and (2.10) do not have explicit solutions. By expanding $h\left(z_{j}\right)=e^{z_{i}}$ in Taylor series keeping only the first 2 terms around

$$
\xi_{j}=F_{Z}^{-1}\left(p_{j}\right)=\ln \left(-\ln q_{j}\right), \quad q_{j}=1-p_{j},
$$

for some $p_{j}$ defined later, we approximate

$$
h\left(z_{j}\right)=e^{z_{j}} \cong h\left(\xi_{j}\right)+h^{\prime}\left(\xi_{j}\right)\left(z_{j}-\xi_{j}\right) \equiv a_{j}+b_{j} z_{j}
$$

with $a_{j}=\left(-\ln q_{j}\right)\left(1-\ln \left(-\ln q_{j}\right)\right), b_{j}=-\ln q_{j}$. Substituting (2.12) and $z_{j}=\left(y_{j}-\mu\right) / \sigma$ into (2.9) and (2.10), we have

$$
\begin{gathered}
\sigma\left[\sum_{i=1}^{n} a_{i}-n_{u}\right]+\sum_{i=1}^{n} b_{i} y_{i}=\mu \sum_{i=1}^{n} b_{i}, \\
\sigma^{2} n_{u}+\sigma\left[\sum_{i=1}^{n} \delta_{i}\left(y_{i}-\mu\right)-\sum_{i=1}^{n} a_{i}\left(y_{i}-\mu\right)\right]-\sum_{i=1}^{n} b_{i}\left(y_{i}-\mu\right)^{2}=0 .
\end{gathered}
$$

From (2.13), we obtain the approximate solution $\tilde{\mu}$ as

$$
\tilde{\mu}=A+B \tilde{\sigma}
$$

with $A=\sum_{i=1}^{n} b_{i} y_{i} / \sum_{i=1}^{n} b_{i}$ and $B=\left(\sum_{i=1}^{n} a_{i}-n_{u}\right) / \sum_{i=1}^{n} b_{i}$. Substituting (2.15) into (2.14), we get the quadratic equation

$$
C \sigma^{2}-D \sigma-E=0 \quad \text { or } \quad n_{u} \sigma^{2}-D \sigma-E=0
$$

where

$$
\begin{aligned}
C & =n_{u}-B\left[n_{u}-\sum_{i=1}^{n} a_{i}+B \sum_{i=1}^{n} b_{i}\right]=n_{u}, \\
D & =\sum_{i=1}^{n} a_{i}\left(y_{i}-A\right)-2 B \sum_{i=1}^{n} b_{i}\left(y_{i}-A\right)-\sum_{i=1}^{n} \delta_{i}\left(y_{i}-A\right), \\
E & =\sum_{i=1}^{n} b_{i}\left(y_{i}-A\right)^{2}>0 .
\end{aligned}
$$


Since $b_{j}=-\ln q_{j}>0$ and $E>0$,

$$
\tilde{\sigma}=\frac{D+\sqrt{D^{2}+4 n_{u} E}}{2 n_{u}}
$$

is the only positive root of the quadratic equation (2.16), and it can be the approximate MLE of $\sigma$. The approximate MLEs of $\alpha$ and $\lambda$ can be $\tilde{\alpha}=1 / \tilde{\sigma}$ and $\tilde{\lambda}=\lambda(\tilde{\alpha})$ from (2.4).

Let us think about the plotting position $p_{j}$ in (2.11). For randomly censored data, we can use the Kaplan-Meier estimator

$$
p_{j}^{K M}=1-\prod_{i \leq j}\left(\frac{n-i}{n-i+1}\right)^{\delta_{i}}
$$

which has been studied in Kaplan and Meier (1958), Efron (1967), Breslow and Crowley (1974), and Meier (1975). The modified one is

$$
p_{j}^{(1)}=1-\prod_{i \leq j}\left(\frac{n-i+1}{n-i+2}\right)^{\delta_{i}}\left(\frac{n}{n+1}\right)^{1-\delta_{1}} .
$$

We put the part $(n /(n+1))^{1-\delta_{1}}$ on the similar quantile probabilities proposed by Herd (1960) and Johnson (1964) to avoid 0 values. Another simple choice is the usual Weibull plotting position $p_{j}^{(2)}=$ $j /(n+1)$. Note that $p_{j}^{(1)}$ reduce to $p_{j}^{(2)}=j /(n+1)$ for a complete sample. We call $\tilde{\sigma}_{1}, \tilde{\alpha}_{1}, \tilde{\lambda}_{1}$ for the approximate MLEs when we use $p_{j}^{(1)}$ in (2.17) and $\tilde{\sigma}_{2}, \tilde{\alpha}_{2}, \tilde{\lambda}_{2}$ when $p_{j}^{(2)}=j /(n+1)$ is used.

\subsection{MLEs and the approximate MLEs under the Koziol-Green model}

If we assume the Koziol-Green model (1.2) in the case that the lifetime distribution follows Weibull $(\lambda$, $\alpha$ ), the censoring distribution also follows a Weibull distribuion with the same shape parameter $\alpha$. Let us assume $G$ follows Weibull $(\eta, \alpha)$, then we have the censoring parameter $\beta=\eta / \lambda$ and the expected proportion of the censored data $\eta /(\lambda+\eta)$. The likelihood function in (2.1) still gives the MLE of $\lambda$ as in (2.4) and the equation of $\alpha$ as

$$
\frac{1}{\alpha}=\frac{\sum_{i=1}^{n} x_{i}^{\alpha} \ln x_{i}}{\sum_{i=1}^{n} x_{i}^{\alpha}}-\frac{1}{n} \sum_{i=1}^{n} \ln x_{i},
$$

which is also the likelihood equation of $\alpha$ for a complete sample. We can obtain the likelihood equation (2.18) from the fact that the distribution of $X_{j}=\min \left(T_{j}, C_{j}\right)$ follows Weibull $(\lambda+\eta, \alpha)$.

As for the approximate MLE of $\alpha$, we use the extreme value distribution $\operatorname{EV}(\mu, \sigma)$ in (2.6) with $\mu=-(1 / \alpha) \ln (\lambda+\eta), \sigma=1 / \alpha$. The same approach in Section 2.1 gives

$$
\tilde{\mu}=A+B \tilde{\sigma}
$$

and the quadratic equation in $\sigma$

$$
n \sigma^{2}-D \sigma-E=0,
$$


Table 1: Averages of the MLE $\hat{\alpha}$ and the approximate MLEs $\tilde{\alpha}_{1}, \tilde{\alpha}_{2}$, MSEs, differences between the MLE and the approximate MLE and RE of two estimates when $\alpha=2, \lambda=0.4,0.6,0.8$ under the assumption that the censoring distribution does not involve any parameters

\begin{tabular}{cc|ccc|ccc|cc|cc}
\hline \hline$\lambda$ & $n$ & $\hat{\alpha}$ & $\tilde{\alpha}_{1}$ & $\tilde{\alpha}_{2}$ & $\operatorname{MSE}(\hat{\alpha})$ & $\operatorname{MSE}\left(\tilde{\alpha}_{1}\right)$ & $\operatorname{MSE}\left(\tilde{\alpha}_{2}\right)$ & $\operatorname{mean}\left(\hat{\alpha}-\tilde{\alpha}_{1}\right)$ & $\operatorname{mean}\left(\hat{\alpha}-\tilde{\alpha}_{2}\right)$ & $\operatorname{RE}\left(\tilde{\alpha}_{1}, \hat{\alpha}\right)$ & $\operatorname{RE}\left(\tilde{\alpha}_{2}, \hat{\alpha}\right)$ \\
\hline 0.4 & 20 & 2.285 & 2.129 & 2.034 & 0.666 & 0.375 & 0.238 & 0.155 & 0.251 & 56.243 & 35.738 \\
& 30 & 2.153 & 2.108 & 2.023 & 0.316 & 0.242 & 0.151 & 0.045 & 0.130 & 76.709 & 47.707 \\
& 40 & 2.110 & 2.062 & 1.997 & 0.225 & 0.169 & 0.105 & 0.047 & 0.112 & 75.040 & 46.785 \\
& 50 & 2.092 & 2.074 & 2.012 & 0.152 & 0.145 & 0.086 & 0.018 & 0.079 & 95.276 & 56.290 \\
\hline 0.6 & 20 & 2.193 & 2.136 & 2.079 & 0.345 & 0.287 & 0.224 & 0.056 & 0.114 & 83.332 & 64.858 \\
& 30 & 2.141 & 2.110 & 2.066 & 0.208 & 0.173 & 0.139 & 0.031 & 0.075 & 83.006 & 66.802 \\
& 40 & 2.095 & 2.078 & 2.041 & 0.133 & 0.123 & 0.099 & 0.017 & 0.054 & 92.247 & 74.165 \\
& 50 & 2.088 & 2.079 & 2.047 & 0.106 & 0.098 & 0.078 & 0.009 & 0.041 & 92.162 & 73.708 \\
\hline 0.8 & 20 & 2.168 & 2.146 & 2.121 & 0.234 & 0.214 & 0.196 & 0.023 & 0.047 & 91.435 & 83.964 \\
& 30 & 2.102 & 2.091 & 2.073 & 0.136 & 0.130 & 0.121 & 0.010 & 0.029 & 95.579 & 88.635 \\
& 40 & 2.080 & 2.076 & 2.061 & 0.100 & 0.097 & 0.091 & 0.004 & 0.019 & 97.166 & 90.474 \\
& 50 & 2.071 & 2.062 & 2.049 & 0.077 & 0.077 & 0.072 & 0.009 & 0.022 & 99.128 & 93.077 \\
\hline \hline
\end{tabular}

MLE = maximum likelihood estimator, $\mathrm{RE}=$ relative efficiency, MSE = mean squared error

where

$$
\begin{aligned}
A & =\frac{\sum_{i=1}^{n} b_{i} y_{i}}{\sum_{i=1}^{n} b_{i}}, \\
B & =\frac{\sum_{i=1}^{n} a_{i}-n}{\sum_{i=1}^{n} b_{i}}, \\
D & =\sum_{i=1}^{n} a_{i}\left(y_{i}-A\right)-2 B \sum_{i=1}^{n} b_{i}\left(y_{i}-A\right)-\sum_{i=1}^{n}\left(y_{i}-A\right), \\
E & =\sum_{i=1}^{n} b_{i}\left(y_{i}-A\right)^{2}>0,
\end{aligned}
$$

and $a_{j}=\left(-\ln q_{j}\right)\left(1-\ln \left(-\ln q_{j}\right)\right), b_{j}=-\ln q_{j}, q_{j}=1-p_{j}, p_{j}=i /(n+1)$. Therefore the approximate MLE of $\sigma$ becomes the positive root of the equation (2.19),

$$
\tilde{\sigma}=\frac{D+\sqrt{D^{2}+4 n E}}{2 n}
$$

and $\tilde{\alpha}=1 / \tilde{\sigma}$.

\section{Simulation results}

In this section, a simulation study is conducted to compare the performance of the MLEs with the approximate MLEs in terms of biases and mean squared errors (MSEs) under the different assumptions of the censoring model. Random samples are generated when the true model is the Koziol-Green model in (1.2). We use the sample sizes $n=20,30,40,50$ and the parameter values $\alpha=2,0.5$, $(\lambda, \eta)=(0.4,0.6),(0.6,0.4),(0.8,0.2)$, which give the proportion of the censoring data $0.6,0.4,0.2$, respectively. Tables 1-4 provide averages of the MLEs and the approximate MLEs, their MSEs, differences between them, and relative efficiency (RE) for the parameters $\alpha$ and $\lambda$, respectively, under the assumption that the censoring distribution does not involve any parameters. Tables 5-8 provide the same statistics under the Koziol-Green model. We replicate each process 2,000 times. The RE, 
Table 2: Averages of the MLE $\hat{\lambda}$ and the approximate MLEs $\tilde{\lambda}_{1}, \tilde{\lambda}_{2}$, MSEs, differences between the MLE and the approximate MLE and RE of two estimates when $\alpha=2, \lambda=0.4,0.6,0.8$ under the assumption that the censoring distribution does not involve any parameters

\begin{tabular}{cc|ccc|ccc|cc|cc}
\hline \hline$\lambda$ & $n$ & $\hat{\lambda}$ & $\tilde{\lambda}_{1}$ & $\tilde{\lambda}_{2}$ & $\operatorname{MSE}(\hat{\lambda})$ & $\operatorname{MSE}\left(\tilde{\lambda}_{1}\right)$ & $\operatorname{MSE}\left(\tilde{\lambda}_{2}\right)$ & $\operatorname{mean}\left(\hat{\lambda}-\tilde{\lambda}_{1}\right)$ & $\operatorname{mean}\left(\hat{\lambda}-\tilde{\lambda}_{2}\right)$ & $\operatorname{RE}\left(\tilde{\lambda}_{1}, \hat{\lambda}\right)$ & $\operatorname{RE}\left(\tilde{\lambda}_{2}, \hat{\lambda}\right)$ \\
\hline 0.4 & 20 & 0.407 & 0.416 & 0.424 & 0.026 & 0.025 & 0.024 & -0.010 & -0.017 & 95.312 & 92.540 \\
& 30 & 0.403 & 0.406 & 0.414 & 0.017 & 0.017 & 0.016 & -0.003 & -0.011 & 97.566 & 94.282 \\
& 40 & 0.404 & 0.407 & 0.413 & 0.012 & 0.011 & 0.011 & -0.003 & -0.009 & 97.588 & 94.681 \\
& 50 & 0.399 & 0.400 & 0.406 & 0.010 & 0.009 & 0.009 & -0.001 & -0.007 & 99.598 & 95.153 \\
\hline 0.6 & 20 & 0.616 & 0.621 & 0.628 & 0.042 & 0.041 & 0.040 & -0.006 & -0.012 & 97.853 & 95.309 \\
& 30 & 0.609 & 0.612 & 0.618 & 0.025 & 0.024 & 0.024 & -0.003 & -0.009 & 97.631 & 95.252 \\
& 40 & 0.611 & 0.613 & 0.618 & 0.019 & 0.019 & 0.018 & -0.002 & -0.007 & 98.324 & 96.440 \\
& 50 & 0.607 & 0.608 & 0.612 & 0.015 & 0.015 & 0.015 & -0.001 & -0.005 & 99.375 & 97.387 \\
\hline 0.8 & 20 & 0.827 & 0.830 & 0.833 & 0.108 & 0.108 & 0.108 & -0.002 & -0.006 & 99.854 & 100.396 \\
& 30 & 0.814 & 0.815 & 0.818 & 0.081 & 0.081 & 0.082 & -0.001 & -0.004 & 99.926 & 100.799 \\
& 40 & 0.813 & 0.813 & 0.816 & 0.069 & 0.070 & 0.070 & 0.000 & -0.003 & 100.201 & 101.267 \\
& 50 & 0.808 & 0.809 & 0.811 & 0.062 & 0.062 & 0.063 & -0.001 & -0.003 & 100.837 & 101.928 \\
\hline \hline
\end{tabular}

$\mathrm{MLE}=$ maximum likelihood estimator, $\mathrm{MSE}=$ mean squared error, $\mathrm{RE}=$ relative efficiency.

Table 3: Averages of the MLE $\hat{\alpha}$ and the approximate MLEs $\tilde{\alpha}_{1}, \tilde{\alpha}_{2}$, MSEs, differences between the MLE and the approximate MLE and RE of two estimates when $\alpha=0.5, \lambda=0.4,0.6,0.8$ under the assumption that the censoring distribution does not involve any parameters

\begin{tabular}{cc|ccc|ccc|cc|cc}
\hline \hline$\lambda$ & $n$ & $\hat{\alpha}$ & $\tilde{\alpha}_{1}$ & $\tilde{\alpha}_{2}$ & $\operatorname{MSE}(\hat{\alpha})$ & $\operatorname{MSE}\left(\tilde{\alpha}_{1}\right)$ & $\operatorname{MSE}\left(\tilde{\alpha}_{2}\right)$ & $\operatorname{mean}\left(\hat{\alpha}-\tilde{\alpha}_{1}\right)$ & $\operatorname{mean}\left(\hat{\alpha}-\tilde{\alpha}_{2}\right)$ & $\operatorname{RE}\left(\tilde{\alpha}_{1}, \hat{\alpha}\right)$ & $\operatorname{RE}\left(\tilde{\alpha}_{2}, \hat{\alpha}\right)$ \\
\hline 0.4 & 20 & 0.560 & 0.528 & 0.503 & 0.040 & 0.023 & 0.014 & 0.032 & 0.057 & 56.973 & 34.912 \\
& 30 & 0.545 & 0.526 & 0.506 & 0.021 & 0.015 & 0.010 & 0.019 & 0.038 & 72.217 & 45.068 \\
& 40 & 0.527 & 0.520 & 0.502 & 0.013 & 0.012 & 0.007 & 0.007 & 0.024 & 86.643 & 52.821 \\
& 50 & 0.522 & 0.515 & 0.500 & 0.010 & 0.009 & 0.005 & 0.008 & 0.023 & 85.355 & 52.461 \\
\hline 0.6 & 20 & 0.549 & 0.535 & 0.521 & 0.023 & 0.017 & 0.014 & 0.014 & 0.028 & 77.586 & 60.953 \\
& 30 & 0.529 & 0.521 & 0.510 & 0.012 & 0.010 & 0.008 & 0.008 & 0.019 & 84.453 & 67.114 \\
& 40 & 0.521 & 0.519 & 0.509 & 0.008 & 0.008 & 0.006 & 0.002 & 0.011 & 90.331 & 72.481 \\
& 50 & 0.514 & 0.513 & 0.505 & 0.006 & 0.006 & 0.005 & 0.001 & 0.010 & 99.539 & 80.320 \\
\hline 0.8 & 20 & 0.539 & 0.535 & 0.529 & 0.015 & 0.015 & 0.013 & 0.004 & 0.010 & 98.553 & 90.588 \\
& 30 & 0.528 & 0.526 & 0.521 & 0.008 & 0.008 & 0.008 & 0.002 & 0.007 & 101.966 & 94.120 \\
& 40 & 0.519 & 0.516 & 0.513 & 0.006 & 0.006 & 0.005 & 0.002 & 0.006 & 94.039 & 87.951 \\
& 50 & 0.513 & 0.512 & 0.509 & 0.005 & 0.004 & 0.004 & 0.001 & 0.004 & 97.638 & 91.554 \\
\hline \hline
\end{tabular}

MLE = maximum likelihood estimator, MSE = mean squared error, RE = relative efficiency.

for example, between two estimates $\tilde{\alpha}, \hat{\alpha}$ is defined as

$$
\operatorname{RE}(\tilde{\alpha}, \hat{\alpha})=\frac{\operatorname{MSE}(\tilde{\alpha})}{\operatorname{MSE}(\hat{\alpha})} \times 100 .
$$

From Tables 1-4, where the estimation process is conducted under the assumption that the censoring distribution does not involve any parameters, we observe the following. First, the approximate MLEs $\tilde{\alpha}_{1}, \tilde{\lambda}_{1}$ using the modified Kaplan-Meier estimator (2.17) give the closer values to the MLEs $\hat{\alpha}, \hat{\lambda}$ on average than the approximate MLEs $\tilde{\alpha}_{2}, \tilde{\lambda}_{2}$ with $p_{j}^{(2)}=j /(n+1)$ for all censoring ratios and sample sizes considered. This phenomena happen for both parameters $\alpha$ and $\lambda$. Second, the estimators $\tilde{\alpha}_{2}, \tilde{\lambda}_{2}$ have the smallest MSEs, and the MLEs $\hat{\alpha}, \hat{\lambda}$ have the largest MSEs for almost all cases. The MSEs are almost the same as for parameter $\lambda$, and usually the MSEs of the MLE $\hat{\alpha}$ are significantly bigger than the MSEs of the approximate MLEs as for parameter $\alpha$. We can also see that the REs between the approximate MLEs and the MLEs tend to decrease as the censoring ratio increases $(\lambda$ decreases) or the sample size decrease. 
Table 4: Averages of the MLE $\hat{\lambda}$ and the approximate MLEs $\tilde{\lambda}_{1}, \tilde{\lambda}_{2}$, MSEs, differences between the MLE and the approximate MLE and RE of two estimates when $\alpha=0.5, \lambda=0.4,0.6,0.8$ under the assumption that the censoring distribution does not involve any parameters

\begin{tabular}{cc|ccc|ccc|cc|cc}
\hline \hline$\lambda$ & $n$ & $\hat{\lambda}$ & $\tilde{\lambda}_{1}$ & $\tilde{\lambda}_{2}$ & $\operatorname{MSE}(\hat{\lambda})$ & $\operatorname{MSE}\left(\tilde{\lambda}_{1}\right)$ & $\operatorname{MSE}\left(\tilde{\lambda}_{2}\right)$ & $\operatorname{mean}\left(\hat{\lambda}-\tilde{\lambda}_{1}\right)$ & $\operatorname{mean}\left(\hat{\lambda}-\tilde{\lambda}_{2}\right)$ & $\operatorname{RE}\left(\tilde{\lambda}_{1}, \hat{\lambda}\right)$ & $\operatorname{RE}\left(\tilde{\lambda}_{2}, \hat{\lambda}\right)$ \\
\hline 0.4 & 20 & 0.404 & 0.411 & 0.419 & 0.027 & 0.026 & 0.025 & -0.007 & -0.015 & 96.387 & 93.325 \\
& 30 & 0.402 & 0.407 & 0.414 & 0.017 & 0.016 & 0.016 & -0.006 & -0.012 & 93.121 & 90.685 \\
& 40 & 0.404 & 0.405 & 0.412 & 0.013 & 0.012 & 0.012 & -0.002 & -0.008 & 97.581 & 93.924 \\
& 50 & 0.405 & 0.408 & 0.414 & 0.010 & 0.009 & 0.009 & -0.003 & -0.008 & 95.671 & 93.205 \\
\hline 0.6 & 20 & 0.616 & 0.622 & 0.628 & 0.089 & 0.089 & 0.091 & -0.005 & -0.012 & 100.767 & 102.439 \\
& 30 & 0.610 & 0.614 & 0.620 & 0.070 & 0.071 & 0.073 & -0.004 & -0.010 & 101.851 & 104.216 \\
& 40 & 0.608 & 0.609 & 0.614 & 0.062 & 0.062 & 0.064 & 0.000 & -0.005 & 99.876 & 102.249 \\
& 50 & 0.605 & 0.606 & 0.610 & 0.056 & 0.056 & 0.058 & -0.001 & -0.005 & 100.045 & 102.423 \\
\hline 0.8 & 20 & 0.838 & 0.840 & 0.843 & 0.249 & 0.251 & 0.253 & -0.002 & -0.005 & 100.629 & 101.450 \\
& 30 & 0.816 & 0.817 & 0.820 & 0.208 & 0.209 & 0.211 & -0.001 & -0.004 & 100.506 & 101.394 \\
& 40 & 0.813 & 0.814 & 0.816 & 0.195 & 0.196 & 0.198 & -0.001 & -0.004 & 100.410 & 101.269 \\
& 50 & 0.814 & 0.814 & 0.816 & 0.190 & 0.191 & 0.192 & 0.000 & -0.003 & 100.053 & 100.850 \\
\hline
\end{tabular}

$\mathrm{MLE}=$ maximum likelihood estimator, $\mathrm{MSE}=$ mean squared error, $\mathrm{RE}=$ relative efficiency.

Table 5: Averages of the MLE $\hat{\alpha}$ and the approximate MLE $\tilde{\alpha}$, MSEs, differences between the MLE and the approximate MLE and RE of two estimates when $\alpha=2, \lambda=0.4,0.6,0.8$ under the Koziol-Green model

\begin{tabular}{cc|cc|cc|cr}
\hline \hline$\lambda$ & $n$ & $\hat{\alpha}$ & $\tilde{\alpha}$ & $\operatorname{MSE}(\hat{\alpha})$ & $\operatorname{MSE}(\tilde{\alpha})$ & $\operatorname{mean}(\hat{\alpha}-\tilde{\alpha})$ & $\operatorname{RE}(\tilde{\alpha}, \hat{\alpha})$ \\
\hline 0.4 & 20 & 2.152 & 2.143 & 0.196 & 0.192 & 0.008 & 97.737 \\
& 30 & 2.103 & 2.100 & 0.114 & 0.113 & 0.003 & 98.783 \\
& 40 & 2.065 & 2.063 & 0.076 & 0.076 & 0.002 & 99.039 \\
& 50 & 2.064 & 2.063 & 0.059 & 0.059 & 0.001 & 100.314 \\
\hline 0.6 & 20 & 2.143 & 2.135 & 0.178 & 0.175 & 0.008 & 98.278 \\
& 30 & 2.112 & 2.107 & 0.113 & 0.111 & 0.004 & 98.351 \\
& 40 & 2.075 & 2.073 & 0.076 & 0.076 & 0.002 & 99.309 \\
& 50 & 2.075 & 2.074 & 0.062 & 0.062 & 0.001 & 100.179 \\
\hline 0.8 & 20 & 2.154 & 2.146 & 0.181 & 0.177 & 0.008 & 97.821 \\
& 30 & 2.096 & 2.092 & 0.108 & 0.107 & 0.004 & 99.238 \\
& 40 & 2.078 & 2.076 & 0.079 & 0.079 & 0.002 & 99.509 \\
\multicolumn{7}{l}{} \\
\hline \hline
\end{tabular}

MLE = maximum likelihood estimator, MSE = mean squared error, RE = relative efficiency.

Table 6: Averages of the MLE $\hat{\lambda}$ and the approximate MLEs $\tilde{\lambda}$, MSEs, differences between the MLE and the approximate MLE and RE of two estimates when $\alpha=2, \lambda=0.4,0.6,0.8$ under the Koziol-Green model

\begin{tabular}{cc|cc|cc|rr}
\hline \hline$\lambda$ & $n$ & $\hat{\lambda}$ & $\tilde{\lambda}$ & $\operatorname{MSE}(\hat{\lambda})$ & $\operatorname{MSE}(\tilde{\lambda})$ & $\operatorname{mean}(\hat{\lambda}-\tilde{\lambda})$ & $\operatorname{RE}(\tilde{\lambda}, \hat{\lambda})$ \\
\hline 0.4 & 20 & 0.418 & 0.419 & 0.024 & 0.024 & -0.0003 & 99.791 \\
& 30 & 0.410 & 0.410 & 0.016 & 0.016 & -0.0001 & 99.830 \\
& 40 & 0.408 & 0.409 & 0.011 & 0.011 & -0.0001 & 99.971 \\
& 50 & 0.402 & 0.402 & 0.009 & 0.009 & 0.0000 & 100.001 \\
\hline 0.6 & 20 & 0.623 & 0.624 & 0.041 & 0.041 & -0.0005 & 99.776 \\
& 30 & 0.614 & 0.615 & 0.024 & 0.024 & -0.0003 & 99.850 \\
& 40 & 0.615 & 0.615 & 0.018 & 0.018 & -0.0001 & 99.881 \\
& 50 & 0.609 & 0.609 & 0.015 & 0.015 & 0.0000 & 99.970 \\
\hline 0.8 & 20 & 0.831 & 0.831 & 0.108 & 0.108 & -0.0006 & 100.030 \\
& 30 & 0.816 & 0.816 & 0.081 & 0.081 & -0.0004 & 100.123 \\
& 40 & 0.814 & 0.814 & 0.070 & 0.070 & -0.0002 & 100.077 \\
& 50 & 0.810 & 0.810 & 0.062 & 0.062 & 0.0000 & 100.020 \\
\hline \hline
\end{tabular}

MLE = maximum likelihood estimator, MSE = mean squared error, RE = relative efficiency. 
Table 7: Averages of the MLE $\hat{\alpha}$ and the approximate MLE $\tilde{\alpha}$, MSEs, differences between the MLE and the approximate MLE and RE of two estimates when $\alpha=0.5, \lambda=0.4,0.6,0.8$ under the Koziol-Green model

\begin{tabular}{cc|cc|cc|cc}
\hline \hline$\lambda$ & $n$ & $\hat{\alpha}$ & $\tilde{\alpha}$ & $\operatorname{MSE}(\hat{\alpha})$ & $\operatorname{MSE}(\tilde{\alpha})$ & $\operatorname{mean}(\hat{\alpha}-\tilde{\alpha})$ & $\mathrm{RE}(\tilde{\alpha}, \hat{\alpha})$ \\
\hline 0.4 & 20 & 0.533 & 0.531 & 0.011 & 0.011 & 0.002 & 99.037 \\
& 30 & 0.525 & 0.524 & 0.007 & 0.007 & 0.001 & 98.778 \\
& 40 & 0.518 & 0.518 & 0.005 & 0.005 & 0.000 & 99.569 \\
& 50 & 0.513 & 0.513 & 0.003 & 0.003 & 0.000 & 99.698 \\
\hline 0.6 & 20 & 0.538 & 0.536 & 0.011 & 0.011 & 0.002 & 98.230 \\
& 30 & 0.521 & 0.520 & 0.006 & 0.006 & 0.001 & 99.288 \\
& 40 & 0.517 & 0.516 & 0.005 & 0.005 & 0.000 & 99.520 \\
& 50 & 0.511 & 0.511 & 0.004 & 0.004 & 0.000 & 100.309 \\
\hline 0.8 & 20 & 0.536 & 0.534 & 0.012 & 0.012 & 0.002 & 98.801 \\
& 30 & 0.526 & 0.525 & 0.007 & 0.007 & 0.001 & 98.819 \\
& 40 & 0.517 & 0.516 & 0.005 & 0.005 & 0.001 & 99.561 \\
\multicolumn{7}{l}{} \\
\hline \hline
\end{tabular}

MLE = maximum likelihood estimator, MSE = mean squared error, RE = relative efficiency.

Table 8: Averages of the MLE $\hat{\lambda}$ and the approximate MLEs $\tilde{\lambda}$, MSEs, differences between the MLE and the approximate MLE and RE of two estimates when $\alpha=0.5, \lambda=0.4,0.6,0.8$ under the Koziol-Green model

\begin{tabular}{cc|cc|cc|rr}
\hline \hline$\lambda$ & $n$ & $\hat{\lambda}$ & $\tilde{\lambda}$ & $\operatorname{MSE}(\hat{\lambda})$ & $\operatorname{MSE}(\tilde{\lambda})$ & $\operatorname{mean}(\hat{\lambda}-\tilde{\lambda})$ & $\operatorname{RE}(\tilde{\lambda}, \hat{\lambda})$ \\
\hline 0.4 & 20 & 0.413 & 0.414 & 0.025 & 0.025 & -0.0004 & 99.877 \\
& 30 & 0.410 & 0.410 & 0.016 & 0.016 & -0.0002 & 99.951 \\
& 40 & 0.408 & 0.408 & 0.012 & 0.012 & -0.0001 & 100.029 \\
& 50 & 0.410 & 0.410 & 0.009 & 0.009 & 0.0000 & 99.977 \\
\hline 0.6 & 20 & 0.623 & 0.624 & 0.090 & 0.090 & -0.0004 & 100.069 \\
& 30 & 0.616 & 0.616 & 0.072 & 0.072 & -0.0003 & 100.077 \\
& 40 & 0.611 & 0.611 & 0.063 & 0.063 & -0.0001 & 100.071 \\
& 50 & 0.608 & 0.608 & 0.057 & 0.057 & 0.0000 & 100.006 \\
\hline 0.8 & 20 & 0.841 & 0.842 & 0.251 & 0.252 & -0.0007 & 100.168 \\
& 30 & 0.818 & 0.819 & 0.210 & 0.210 & -0.0004 & 100.114 \\
& 40 & 0.814 & 0.815 & 0.196 & 0.196 & -0.0002 & 100.078 \\
& 50 & 0.815 & 0.815 & 0.191 & 0.191 & -0.0001 & 100.028 \\
\hline \hline
\end{tabular}

MLE = maximum likelihood estimator, MSE = mean squared error, RE = relative efficiency.

From Tables 5-8, where the estimation is conducted under the true model (the Koziol-Green model in (1.2)), the average differences between the MLEs and the approximate MLEs are negligible. Apparently the MSEs depend on sample sizes rather than censoring ratios. The assumption of the censoring distribution has a strong influence on the estimation of $\alpha$ rather than that of $\lambda$, especially when the censoring ratio is big.

\section{Concluding remarks}

In this paper, we study randomly censored data from a Weibull distribution. We derive the approximate MLEs in explicit forms using the type I extreme value distribution of the minimum since the MLEs of the parameters do not have explicit solutions. In the simulation study, we generate random samples under the Koziol-Green model, compute the estimators ignoring the censoring model, and compare them with the estimators from the true model. Consequently, we find that the censoring model has a strong influence on the scale parameter $\alpha$ rather than the shape parameter $\lambda$. The MSE of the scale parameter becomes larger when we ignore the censoring model. The approximate MLEs are quite similar to the MLEs in terms of biases and MSEs in any situation. 


\section{References}

Balakrishnan N and Kateri M (2008). On the maximum likelihood estimation of parameters of Weibull distribution based on complete and censored data, Statistics \& Probability Letters, 78, 29712975.

Breslow N and Crowley J (1974). A large sample study of the life table and product limit estimates under random censorships, Annals of Statistics, 2, 437-453.

Chen YY, Hollander M, and Langberg NA (1982). Small-sample results for the Kaplan-Meier estimator, Journal of the American Statistical Association, 77, 141-144.

Chen Z (1998). Joint estimation for the parameters of the extreme value distributions, Statistical Papers, 39, 135-146.

Csörgó S and Horváth L (1981). On the Koziol-Green model for random censorship, Biometrika, 68 , 391-401.

Efron B (1967). The two sample problem with censored data. In Proceedings of the 5th Berkeley Symposium on Mathematical Statistics and Probability, Berkeley, CA, 831-853.

Engelhardt M and Bain LJ (1974). Some results on point estimation for the two-parameter Weibull or extreme-value distribution, Technometrics, 16, 49-56.

Herd GR (1960). Estimation of reliability from incomplete data. In Proceedings of the 6th National Symposium on Reliability and Quality Control, Washington, DC, 202-217.

Johnson LG (1964). The Statistical Treatment of Fatigue Experiments, Elsevier, Amsterdam.

Kang SB and Han JT (2009). Goodness-of-fit test for the Weibull distribution based on multiply typeII censored samples, Communications for Statistical Applications and Methods, 16, 349-361.

Kaplan EL and Meier P (1958). Nonparametric estimation from incomplete observations, Journal of the American Statistical Association, 53, 457-481.

Kim N (2014). Approximate MLE for the scale parameter of the generalized exponential distribution under random censoring, Journal of the Korean Statistical Society, 43, 119-131.

Koziol JA and Green SB (1976). A Cramér-von Mises statistic for randomly censored data, Biometrika, 63, 465-474.

Kundu D (2007). On hybrid censored Weibull distribution, Journal of Statistical Planning and Inference, 137, 2127-2142.

Meier P (1975). Estimation of a distribution function from incomplete observations. In J. Gani (Ed), Perspectives in Probability and Statistics (pp. 67-87), Academic Press, New York.

Pareek B, Kundu D, and Kumar S (2009). On progressively censored competing risks data for Weibull distributions, Computational Statistics \& Data Analysis, 53, 4083-4094.

Tableman M and Kim JS (2003). Survival Analysis Using S: Analysis of Time-to-Event Data, CRC Press, Boca Raton, FL. 